\title{
Das „Recht auf Vergessenwerden“
}

Das „Recht auf Vergessenwerden“ stellt ein Betroffenenrecht dar und ist in der DS-GVO in Art. 17 Abs. 2 normiert. ${ }^{1}$ Die Bezeichnung „Recht auf Vergessenwerden“ wirkt auf den ersten Blick als rechtlicher Terminus zumindest merkwürdig, da unter dem Begriff „Vergessenwerden“ ein menschliches Phänomen, nämlich der Verlust von im Gedächtnis gespeicherten Informationen zu verstehen ist, ${ }^{2}$ der Begriff also eine kognitive Fähigkeit betrifft. Das in Art. 17 Abs. 2 DS-GVO vermittelte „Recht auf Vergessenwerden " begründet hingegen eine Informationspflicht, welche dann zur Anwendung kommen soll, wenn ein Anspruch auf Löschung besteht und die zu löschenden Daten öffentlich gemacht wurden. ${ }^{3}$ Die Erklärung für die Verwendung dieser für die juristische Fachsprache untypischen Bezeichnung erschließt sich aus dem Anwendungskontext dieses Rechts, es dient nämlich dem der bloßen Information an Dritte übergeordneten Zweck der Beseitigung von Informationen aus dem Internet, welches bekanntlich „nichts vergisst“. ${ }^{\text {. }}$

Dabei stellt sich der typische Anwendungsfall des „Rechts auf Vergessenwerden“ nach Art. 17 Abs. 2 DS-GVO wie folgt dar: Im Internet veröffentlichte Daten werden häufig von anderen Verantwortlichen gespeichert und teilweise wiederum veröffentlicht, z.B. auf Servern der Organisation „Internet Archive“ (https://archive. org), oder verlinkt, z.B. auf Ergebnisseiten von Suchmaschinen. ${ }^{5}$ Es ist also durchaus möglich und wahrscheinlich, dass Daten auf solchen Seiten weiterhin existieren, obwohl die Daten auf den ursprünglichen Websites gelöscht wurden. ${ }^{6}$ Während die Löschung der Daten auf den ursprünglichen Websites Ziel des Anspruchs aus Art. 17 Abs. 1 DS-GVO ist, verlangt Abs. 2 vom Verantwortlichen, „angemessene Maßnahmen“ zu treffen, um die übrigen Verantwortlichen, welche die Daten weiterhin verarbeiten (z.B. Suchmaschinen), über das Löschungsverlangen der betroffenen Person zu informieren. Auch technische Maßnahmen kommen in Betracht. ${ }^{7}$ Bei der Beurteilung, welche Maßnahmen zur Durchführung der Informationspflicht an die übrigen Verantwortlichen als angemessen anzusehen sind, sind die verfügbare Technologie sowie die Implementierungskosten zu berücksichtigen. Nach Art. 70 Abs. 1 lit. d DS-GVO soll der Europäische Datenschutzausschuss Leitlinien, Empfehlungen und bewährte Verfahren bereitstellen, wodurch die in Art. 17 Abs. 2 DS-GVO enthaltenen Pflichten eine gewisse Konkretisierung erfahren. Diese sind zwar unverbindlich, sie sollen aber bei der Auswahl einer angemessenen Maßnahme jedenfalls berücksichtigt werden. ${ }^{8}$

\footnotetext{
1 Nolte/Werkmeister in Gola, DS-GVO, 1. Aufl. 2017, Art. 17 Rn. 1.

2 Hornung/Hofmann, JZ 2013, 163 (164).

3 Nolte/Werkmeister in Gola, DS-GVO, 1. Aufl. 2017, Art. 17 Rn. 1.

4 Vgl. Herbst in Buchner/ Kühling, DS-GVO, 1. Aufl. 2017, Art. 17 Rn. 49.

5 Ebenda.

6 Ebenda.

7 Hierzu Hornung/Hofmann, JZ 2013, 163 (168 f.).

8 Herbst in Buchner/ Kühling, DS-GVO, 1. Aufl. 2017, Art. 17 Rn. 58.
}

Der nach Art. 17 Abs. 2 DS-GVO Verpflichtete hat über das Löschungsverlangen alle Verantwortlichen zu informieren, die die betreffenden Daten verarbeiten, unabhängig davon, wie sie an die Daten gelangt sind. ${ }^{9}$ Die Rechtsfolge des Art. 17 Abs. 2 DS-GVO geht - anders, als die Überschrift "Recht auf Vergessenwerden“ es vermuten lässt - nicht so weit, dass die übrigen Verantwortlichen die Daten zu löschen haben, sobald sie über das Löschungsbegehren der betroffenen Person vom verantwortlichen Adressaten des Art. 17 Abs. 2 DS-GVO erfahren haben. Und auch Letzterer hat nicht die Pflicht, für die Löschung dieser weiterverarbeiteten Daten zu sorgen. ${ }^{10}$ Eine Pflicht zur Löschung kann sich für die übrigen Verantwortlichen aber wiederum aus Art. 17 Abs. 1 DS-GVO ergeben.

Die Bezeichnung „Recht auf Vergessenwerden“ erlangte aufgrund des „Google Spain“-Urteils des EuGH ${ }^{11}$ aus dem Jahr 2014 erstmals breite Aufmerksamkeit, obwohl die Bezeichnung sich so im Urteil gar nicht findet. Zu beachten ist, dass sowohl das Urteil als auch die Regelung des Art. 17 Abs. 2 DS-GVO zwar dasselbe Ziel vor Augen haben, nämlich bestimmte Informationen aus dem Internet zu beseitigen. Der Weg zur Erreichung dieses Ziels ist aber jeweils ein anderer: ${ }^{12}$ Im EuGH-Urteil ging es um einen Anspruch der betroffenen Person gegen einen Suchmaschinenbetreiber auf Löschung von Daten im Suchmaschinenindex. Indem diese Daten von der Suchmaschine nicht mehr aufgelistet werden durften, sollte deren Auffindbarkeit erschwert werden. Im Unterschied dazu regelt Art. 17 Abs. 2 DS-GVO den Anspruch gegen den für die ursprüngliche Datenverarbeitung Verantwortlichen, Dritte (z.B. Suchmaschinenbetreiber) über das Löschungsverlangen der betroffenen Person zu informieren. Diese zwei Wege zur Verwirklichung des „Rechts auf Vergessenwerden“ bedeuten damit in Bezug aufSuchmaschinenbetreiber, dass diese einerseits im Sinne des Art. 17 Abs. 2 DS-GVO als die durch den ursprünglich Verantwortlichen zu informierenden Dritten anzusehen sind, sie andererseits aber auch im Sinne des EuGH-Urteils als Adressaten eines Anspruchs aus Art. 17 Abs. 1 DS-GVO in Betracht kommen. Der im EuGH-Urteil diskutierte Anspruch auf Löschung wurde vom EuGH bejaht, weil die Grundrechte der betroffenen Person aus Art. 7, 8 GRCh im Vergleich zu den wirtschaftlichen Interessen des Verantwortlichen und dem Interesse der Öffentlichkeit am Zugang zur betreffenden Information überwogen. Insoweit liefert das EuGH-Urteil Anhaltspunkte dafür, unter welchen Voraussetzungen Suchmaschinenbetreiber zur Löschung von Links in Ergebnislisten nach Art. 17 Abs. 1 DS-GVO verpflichtet sind, die dann zur Anwendung kommen, wenn das Vorliegen eines Löschungsrechts von einer Abwägung (wie sie z.B. Art. 6 Abs. 1 lit. f DS-GVO erfordert) abhängt.

\footnotetext{
9 Ebenda Rn. 54.

10 Ebenda Rn. 61

11 EuGH Urt. v. 13.05.2014 - C-131/12, DuD 2014, 559.

12 Herbst in Buchner/ Kühling, DS-GVO, 1. Aufl. 2017, Art. 17 Rn. 67 f.
} 